\title{
Incidência das Formas Imaturas de Aedes albopictus (Skuse) e Aedes aegypti (Linnaeus) no Município de Miguel Pereira, RJ, Brasil
}

\author{
Paulo Roberto Moreira Barbosa1 ${ }^{1}$, William Costa Rodrigues ${ }^{2} \&$ Marise Maleck de Oliveira Cabral $^{3} \bowtie$
}

1. Curso de Ciências Biológicas/Centro de Ciências Exatas, Tecnológicas e da Natureza Universidade Severino Sombra, Vassouras, RJ. e-mail: professorpaullo@gmail.com. 2. Universidade Severino Sombra, FAETEC/Instituto Superior de Tecnologia, Paracambi, RJ e Methodos Consultoria Ambiental. e-mail: wcrodrigues@ebras.bio.br. 3. Curso de Ciências Biológicas/Centro de Ciências Exatas, Tecnológicas e da Natureza/Laboratório de Insetos Vetores/Unidade de Apoio ao Ensino, Pesquisa e Extensão Prof. Antonio O. Izolani, Universidade Severino Sombra/Lab. de Diptera, Inst. Oswaldo Cruz, e-mail: mmaleck@oi.com.br. (Autor para correspondência ${ }^{\bowtie}$ )

\section{EntomoBrasilis 3 (2): 55-58 (2010)}

Resumo. A presença de Aedes albopictus (Skuse) em área urbana representa um risco potencial do inter-relacionamento dessa espécie de mosquito com a população. Este estudo teve como objetivo identificar as formas imaturas de Aedes albopictus (Linnaeus) encontradas nos depósitos e imóveis do município de Miguel Pereira. O estudo foi realizado no período de 12 meses, ano de 2007, em 27 bairros do município de Miguel Pereira. As larvas encontradas foram identificadas no laboratório, e calculados os índices de infestação predial (IIP) e índice de Breteau (IB). Os tipos de imóveis positivos foram residências (79,45 \% do total); terrenos baldios (2,57\%); comércios (2,57\%) e outros tipos de imóveis (15,05\%) para Ae. albopictus ( $\mathrm{IIP}=1,0)$, e residências (72,73\% do total); comércios (6,06\%); terrenos baldios (3,03\%); e outros tipos de imóveis (18,18\%) para Ae. aegypti (IIP= 0,1). Ao todo foram capturadas 3731 larvas, sendo 3562 (95, 47\%) de Ae. albopictus e 169 (4,53\%) de Ae. aegypti. As formas imaturas de Ae. albopictus mostraram-se mais presente que Ae. aegypti no Município de Miguel Pereira no ano de 2007, e podendo futuramente se tornar um problema de saúde pública.

Palavras-chave: Aedes aegypti; Aedes albopictus; Culicidae; Depósitos preferenciais; insetos vetores

\section{Incidence of Aedes albopictus (Skuse) and Aedes aegypti (Linnaeus) immature forms in Miguel Pereira City, RJ, Brazil}

Abstract.The presence of Aedes albopictus (Skuse) in urban areas represents a potential risk of interrelationship of this mosquito species with the population. This study aimed to evaluate the presence of Aedes albopictus (Linnaeus) immature forms in deposits and buildings at Miguel Pereira city, Rio de Janeiro State. The study was carried in the last 12 months, 2007, in 27 districts in Miguel Pereira city. The larvae were identified at the laboratory, and calculated the rates of predial infestation index (IIP) and Breteau index (BI). The positive buildings were: residential (79.45 \% of total); waste land (2.57\%), shopping (2.57\%) and other types of buildings (15.05\%) to Ae. albopictus (PII $=1.0)$ and homes ( $72.73 \%$ of total), shopping (6.06\%); waste land (3.03\%) and other types of buildings (18.18\%) to Ae. aegypti (PII= 0.1) The 3731 larvae collected, 3562 (95.47\%) were Ae. albopictus and 169 (4.53\%) of Ae. aegypti. The immature forms of Ae. albopictus proved to be more present than Ae. aegypti in the Miguel Pereira city in 2007 and in future it may become a public health problem.

Keywords: Aedes aegypti; Aedes albopictus; Culicidae; preferred deposits; vectors insects

$\boldsymbol{A}$ edes albopictus Skuse (Diptera: Culicidae) é um mosquito asiático que teve sua dispersão incrementada para outras partes do mundo desde 1980. Sua primeira identificação no Brasil ocorreu na cidade do Rio de Janeiro, em 1986, e nenhum outro registro anterior é conhecido. Partindo da área primeiramente infestada, essa espécie vem sucessivamente se disseminando pelo Brasil e chegando atingir 20 dos 27 estados da Federação (SAntos 2003; Alves 2008). Esse mosquito tem demonstrado elevada capacidade para utilizar uma ampla variedade de criadouros artificiais no território brasileiro, sem abandonar ecótopos naturais (ForatTini et al. 1995; Gomes et al. 1992; LOURENÇO-De-Oliveira et al., 2004).

As formas imaturas desse mosquito se mantêm, principalmente, em pneus, caixa d'água, vasos de plantas, latas, garrafas, bebedouros de animais e/ou ainda outros objetos que retenham água (Ministério DA SAÚde 1998). Esses criadouros artificiais são encontrados facilmente nas casas e descritos como pontos estratégicos (PE) segundo o Programa Nacional de Controle de Dengue e Febre Amarela (MinistéRIo da SAúde 1998), e geralmente infestados com Ae. albopictus e/ou Aedes aegypti (Linnaeus) (Suwonkerd et al. 1996) sendo considerados um dos mais produtivos depósitos para estes mosquitos.
No contexto da perspectiva de ampliação da dispersão de Ae. albopictus aos ambientes selváticos brasileiros, ricos em populações de vírus associados a doenças humanas, fazse necessária uma análise das condições de risco potencial e do seu envolvimento nos ciclos desses patógenos (Gomes et al. 1999). Sabendo-se das condições favoráveis na manutenção de vetores e patógenos específicos na natureza, conduz à identificação dos riscos temporal e espacial de doenças (SHARma \& SRIVASTAVA,1997).

A população de Ae. albopictus introduzida no Brasil demonstrou, sob condições laboratoriais, a capacidade de transmitir dengue, febre amarela e vírus da encefalite equina venezuelana (MILLER \& BALLINGER 1988). A isto se acrescenta o registro de um único vírus do dengue pertencente ao sorotipo 1 (DEN-1) numa larva de Ae. albopictus de Campos Altos - MG (SERUfo et al. 1993).

A potencialidade vetorial do Ae. albopictus para estados norte-americanos de 22 distintos arbovírus (Moore \& Mitchell 1997), de vetor efetivo do vírus do dengue na Ásia e no Pacífico (Moore \& Mitchell 1997; Alves et al. 2008), da capacitação de dispersão aos ambientes rurais e silvestres, e bom transmissor dos vírus por via transovariana (Alves 2008) justifica identificar 
a sua distribuição, localização, depósitos e criadouros de $A e$. albopictus no Município de Miguel Pereira, RJ.

O objetivo deste estudo foi avaliar a incidência de $A e$. albopictus e Ae. aegypti no Município de Miguel Pereira, RJ, através da positividade de imóveis e depósitos inspecionados.

\section{MATERIAL E MÉTODOS}

Caracterização do local de estudo. O Município de Miguel Pereira (Figura 1) possui uma extensão territorial de 287 km2, clima tropical, 618 metros de altitude, temperatura média de $24^{\circ} \mathrm{C}$ e índice pluviométrico de $1750 \mathrm{~mm}^{3}$ e 24.585 habitantes (IBGE 2007).

Levantamento e coleta. A área de estudo foi dividida em 27 localidades. Para descrever a presença de Ae. albopictus no município de Miguel Pereira foram observados 51.820 imóveis e 180.762 depósitos. O levantamento foi realizado no ano de 2007, por um período consecutivo de doze meses, divididos em ciclos operacionais de dois meses.

Utilizou-se para esse estudo o levantamento de pesquisa de focos larvários que teve como finalidade precípua conhecer a infestação dos vetores e a deteç̧ão de depósitos para o Ae. aegypti e Ae. albopictus (TAVEIRA et al. 2001; Donalísio \& GLASSER 2002).

Os depósitos foram definidos como um grupo e classificados de acordo com o tipo de depósito em letras de A-K (MinISTÉrio DA SAÚDE 1998) e, ao conjunto de larvas capturadas em um mesmo tipo de depósito e acondicionadas em um único tubo (Ministério DA SAÚde 1998) definindo um "pool" representativo a cada depósito. As larvas coletadas por dia foram agregadas, diariamente. A cada coleta os exemplares foram mortos em álcool a 70\%, acondicionados em tubos de hemólise e transportados ao laboratório para a identificação.

A identificação dos espécimes foi realizada pela observação direta dos caracteres morfológicos evidenciáveis ao estereoscópico e ao microscópio de luz transmitida utilizando a chave dicotômica proposta por Forattini (2002). Dentre os espécimes coletados, apenas os culicídeos Ae. aegypti e Ae. albopictus foram notificados como foco positivo, sendo que as outras espécies também foram identificadas. Os dados foram analisados por variância não paramétrica, através de ANOVA e calculados os índices de infestação predial (IIP) e de Breteau
(IB).

\section{RESULTADOS}

Dos 51.820 imóveis inspecionados em 27 bairros, 545 imóveis $(94,5 \%)(\mathrm{IIP}=1,0)$ foram positivos para Ae. albopictus e 33 imóveis $(5,71 \%)($ IIP $=0,1)$ positivos para Ae. aegypti (Tabela 1), e ainda considerando o índice de pendência $(30,36 \%)$ relacionado aos imóveis que não puderam ser notificados. Dentre os imóveis positivos, as residências prevalecem para a presença de ambas as espécies, seguidas do tipo outros (diferentes tipos de imóveis) (Tabela 2). Dos 1099 depósitos positivos prevaleceram as larvas de Ae. albopictus em $95 \%(\mathrm{IB}=1,9)$ comparados com 4,8\% (IB = o,1) de Ae. aegypti (Tabela 3). Dos depósitos predominantes destacaram-se: outros (I), vasos de planta (C), pneu (A), recipiente natural $(\mathrm{H})$, garrafa, lata e plástico (E) (Tabela 4). Os depósitos tipo outros (I) referem-se aos depósitos que não se enquadram em nenhuma das categorias classificáveis (A-K). Os depósitos preferenciais para Ae. aegypti foram os vasos de planta (C) $(32,55 \%)$ seguido das garrafas, latas e plásticos (E) $(30,23 \%)$ e para Ae. albopictus os depósitos preferenciais também foram os vasos de plantas (C) $(32,55 \%)$ seguido do tipo outros (I) em 26,58\% (Tabela 4) . Os depósitos inspecionados, no município em estudo, que se mostraram positivos para ambas as espécies de Aedes foram notificados e imediatamente tratados com os biolarvicidas Vectobac G e Vectobac WG pelo Programa Municipal de Controle da Dengue (PMCD). Neste levantamento foram capturadas 3.731 larvas de Aedes sendo 3562 larvas (95,5 \%) de Ae. albopictus, 169 larvas (4,5 \%) de Ae. aegypti (Tabela 5), e 7.054 larvas de outras espécies de mosquitos no município de Miguel Pereira, RJ.

Tabela 1. Incidência de imóveis positivos para Ae. aegyptie Ae. albopictus no período de 12 meses, no ano de 2007, no município de Miguel Pereira, RJ.

\begin{tabular}{lcccc}
\hline \multirow{2}{*}{ Espécies } & \multicolumn{5}{c}{ Imóveis Positivos } \\
\cline { 2 - 5 } & No Total & $\mathbf{X} \pm \mathbf{D P}$ & $\mathbf{\%}$ & $\mathbf{I I P}$ \\
\hline Ae. aegypti & 33 & $1,2 \pm 1,95$ & 5,71 & 0,1 \\
Ae. albopictus & 545 & $19,8 \pm 24,5^{* * *}$ & 94,29 & 1,0 \\
Z tabelado & - & 1,96 & - & - \\
Z calculado & - & $-4,37$ & - & - \\
\hline
\end{tabular}

*Significativo a $5 \%(\alpha=0,05)$ pela tabela de valores críticos de $\mathrm{Z}$, ANOVA $(\mathrm{N}=27, \mathrm{df}=1)$.

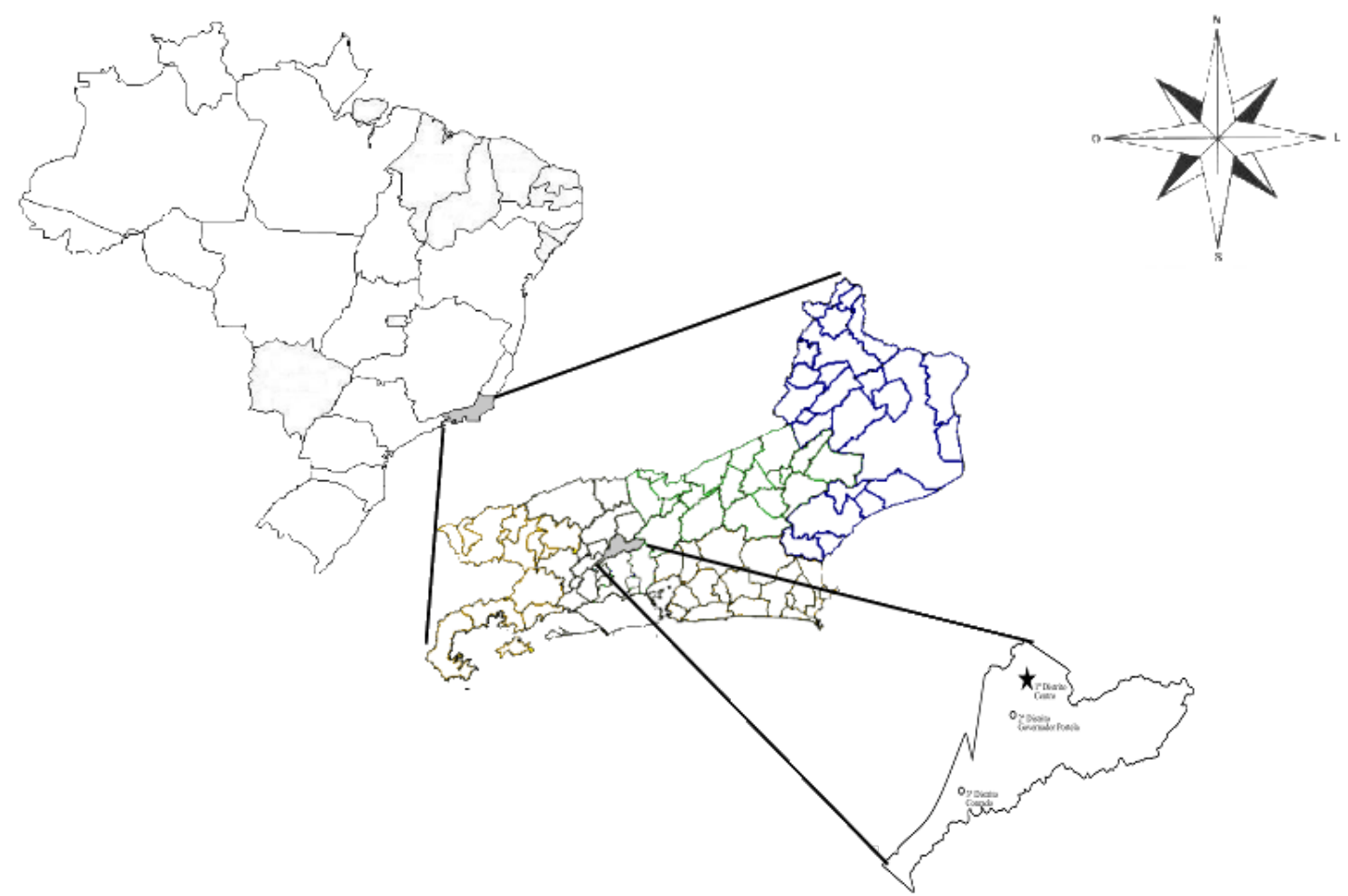

Figura 1. Localização geográfica do município de Miguel Pereira (em destaque), no Estado do Rio de Janeiro, Brasil. 
Tabela 2. Número e tipos de imóveis positivos para Ae. albopictus e Ae aegypti no período de 12 meses, no ano de 2007, no município de Miguel Pereira, RJ.

\begin{tabular}{lcccc}
\hline \multirow{2}{*}{ Tipos de Imóvel } & \multicolumn{2}{c}{ Ae. albopictus } & \multicolumn{2}{c}{ Ae. aegypti } \\
\cline { 2 - 5 } & No & $\mathbf{\%}$ & No & \% \\
\hline Residência & 433 & 79,45 & 24 & 72,73 \\
Comércio & 14 & 2,57 & 02 & 6,06 \\
Terrenos Baldios & 14 & 2,57 & 01 & 3,03 \\
\hline Outros tipos de imóveis & 82 & 15,05 & o6 & 18,18 \\
\hline Pontos Estratégicos & 02 & 0,37 & oo & 0,00 \\
\hline Total & $\mathbf{5 4 5}$ & $\mathbf{1 0 0}$ & $\mathbf{3 3}$ & $\mathbf{1 0 0}$ \\
\hline
\end{tabular}

Tabela 3. Incidência de depósitos positivos para Ae. aegypti e Ae.albopictus no período de 12 meses, no ano de 2007, no município de Miguel Pereira, RJ.

\begin{tabular}{lcccc}
\hline \multirow{2}{*}{ Espécies } & \multicolumn{4}{c}{ Depósitos Positivos } \\
\cline { 2 - 5 } & No Total & $\mathbf{X} \pm$ DP & \% & IB \\
\hline Ae. aegypti & 53 & $2,0 \pm 3,8$ & 4,82 & 0,1 \\
Ae. albopictus & 1046 & $39,8 \pm 52,4^{* * * *}$ & 95,18 & 1,9 \\
Z tabelado & - & 1,96 & - & - \\
Z calculado & - & $-4,55$ & - & - \\
\hline${ }^{*}$ Significativo a $5 \%(\alpha=0,05)$ pela tabela de valores críticos de $\mathrm{Z}$, ANOVA $(\mathrm{N}=27, \mathrm{df}=1)$.
\end{tabular}

Tabela 4. Tipos e números de depósitos para a Ae. albopictus e Ae. aegypti no período de 12 meses no ano de 2007, no município de Miguel Pereira, RJ.

\begin{tabular}{lcccc}
\hline \multirow{2}{*}{ Tipos de Depósito } & \multicolumn{2}{c}{ Ae. albopictus } & \multicolumn{2}{c}{ Ae.aegypti } \\
\cline { 2 - 5 } & No & $\mathbf{\%}$ & No & $\%$ \\
\hline Vaso de planta (C) & 278 & 32,55 & 14 & 32,56 \\
Pneu (A) & 155 & 18,15 & 07 & 16,28 \\
Outros (I) & 227 & 26,58 & 09 & 20,93 \\
$\begin{array}{l}\text { Garrafa, lata } \\
\text { plástico (E) }\end{array}$ & 194 & 22,72 & 13 & 30,23 \\
\hline Total & $\mathbf{8 5 4}$ & $\mathbf{1 0 0}$ & $\mathbf{4 3}$ & $\mathbf{1 0 0}$ \\
\hline
\end{tabular}

Tabela 5. Incidência de formas imaturas de Ae. aegypti e Ae.albopictus no período de 12 meses no ano de 2007, no município de Miguel Pereira, RJ.

\begin{tabular}{lccc}
\hline \multirow{2}{*}{ Espécies } & \multicolumn{3}{c}{ Formas Imaturas } \\
\cline { 2 - 4 } & No Total & $\mathbf{X} \pm \mathbf{D P}$ & \% \\
\hline Ae. Aegypti & 169 & $6,5 \pm 15,9$ & 4,53 \\
Ae. albopictus & 3.562 & $135,9 \pm 186,3^{* * *}$ & 95,47 \\
Total & 3.731 & - & 100 \\
Z tabelado & - & 1,96 & - \\
Z calculado & - & $-4,59$ & - \\
\hline
\end{tabular}

*Significativo a $5 \%(=0,05)$ pela tabela de valores críticos de $\mathrm{Z}$, ANOVA $(\mathrm{N}=27, \mathrm{df}=1)$.

\section{DISCUSSÃO}

O presente estudo revelou a ocorrência das duas espécies de Aedes e, considerou o local como indicação de característica domiciliada para Ae. albopictus. O estudo em questão corrobora com Chiaravalloti et al. (1997) que avaliando a distribuição espacial de Ae. albopictus, detectou sua ocupação nas áreas urbanas, de modo não uniforme, destacando as áreas periféricas como as mais freqüentadas por essa espécie. Esses autores ainda apontaram a presença de vegetação como possível fator favorável à infestação pelo Ae. albopictus. De acordo com ForATTini et al., (1995) a característica de sobrevivência da espécie pode ser uma resposta à sua adaptabilidade nas cidades, principalmente quando essas são bem arborizadas e apresentam uma gama diversificada de micro habitats, características aplicáveis à cidade de presente estudo.

A dificuldade em controlar a proliferação do Ae. albopictus é consequente a ampla distribuição de seus criadouros naturais e artificiais (FrANCO-EstradA \& CRAIG JR 1995; HAWLEY 1988; Lopes 1993). No Brasil, como espécie fitotelmata, suas formas imaturas foram encontradas em ocos de árvores e internódios de bambu (Gomes et al. 1992; Gomes \& MARQues 1988), bromélias (Ferreira Neto et al. 1987; Gonçalves \& Messias 2008; Forattini et al. 1998) e registradas colonizando bambus e pneus em um remanescente da Mata Atlântica da área urbana de Recife, Estado de Pernambuco (Albuquerque et al. 2000). Os autores Gilotra et al. (1967); O'MEARA et al. (1995) e SOAREs et al. (2008), apontaram que na área urbana, o Ae. albopictus utiliza como criadouro os mesmos depósitos artificiais utilizados pelo Ae. aegypti, preferindo porém os situados no peridomicílio. Os dados deste estudo demonstraram 95,18\% de depósitos positivos para Ae. albopictus com o percentual de $26,58 \%$ de sua ocorrência nos depósitos artificiais, corroborando com Consoli \& LouRENÇODe-Oliveira (1994) que apontam os recipientes artificiais abandonados nas florestas e plantações como um dos habitas do Ae. albopictus, e com Gomes et al. (1992) que constataram a presença do mosquito em áreas rurais, urbanas e suburbanas,

A positividade de Ae. albopictus em preferência a Ae. aegypti, nos imóveis e depósitos no município de Miguel Pereira, área urbana mas com características naturais devido a abundante cobertura vegetal, é corroborada com BALESTRA et al. (2008) e UrbinatTi (2004) que apontam a presença frequente de Ae. albopictus tanto em áreas de proteção ambiental como em áreas alteradas, reforçando a evidência de sua ampla valência ecológica em colonizar ecótopos naturais e artificiais.

\section{REFERÊNCIAS}

Albuquerque, C.M.R., M.A.V. Melo-Santos, M.A.S. Bezerra, R.M.R. Barbosa, D.F. Silva \& E. Silva, 2000. Primeiro registro de Aedes albopictus em área de Mata Atlântica, Recife, PE, Brasil. 34. Revista de Saúde Pública, 34: 314-315.

Alves, J.R.C., J. Alencar \& J.M. Costa, 2008. Ocorrência de larvas de Aedes albopictus (Skuse) (Diptera, Culicidae), em recipiente artificial, na ilha de Marambaia, Mangaratiba, RJ, Brasil. Revista de Patologia Tropical, 37 (2): 177-180.

Balestra, R.A.M., R.O. Pereira, M.J.S. Ribeiro, J.S. Silva \& J. Alencar, 2008. Ocorrência de Aedes (Stegomyia) albopictus (Skuse) em Área Urbana do Estado do Tocantins. Neotropical Entomology, 37: $233-235$.

Chiaravalloti Neto, F., 1997. Descrição da colonização de Aedes aegypti na região de São José do Rio Preto, SP. Revista Sociedade brasileira Medicina Tropical, 30: 279 - 285.

Consoli, R.A.G.B. \& Lourenço-de-Oliveira, R., 1994. Principais Mosquitos de Importância Sanitária no Brasil, Fiocruz, Rio de Janeiro.

Donalisio, M.R. \& , C.M. Glasser, 2002. Vigilância Entomológica e Controle de Vetores de Dengue. Revista Brasileira de Entomologia, 5: 260 - 272.

Ferreira Neto, J.A., M.M. Lima \& M.B. Aragão, 1987. Primeiras observações sobre o Ae. albopictus no Estado do Espírito Santo, Brasil. Caderno de Saúde Pública, 3: 56 - 61,

Forattini, O.P., I. Kakitani, E. Massad \& D. MARUCCI, 1995. Studies on mosquitoes (Diptera:Culicidae) and anthropic environment. 9- Synanthropy and epidemiological vector role of Aedes scapularis in south-Eastern Brazil. Revista Saúde Pública, 29: $199-207$.

Forattini, O.P., 1998. Mosquitos Culicidae como vetores emergentes de infecções. Revista de Saúde Pública, 32: 497 -502 .

Forattini, O.P., 2002. Culicidologia Médica. Identificação, Biologia, epidemiologia. Ed. Universidade de São Paulo, Brasil. Vol .2, 864p.

Franco-Estrada, J.G. \& G.B. Craig Jr., 1995. Biologia relaciones con enfermedades y control deAedes albopictus. Organización Panamericana de La Salud. Washington (DC).

Gilotra, S.K., L.E. Rozeboon \& N.C. Bhattacharya, 1967. Observations on possible competitive displacement between populations of Aedes aegypti Linnaeus and Aedes albopictus 
Skuse in Calcutta. Bullettin World Health Organ, 37: 437-46. Gomes A.C., O.P. Forrattini, I. Kakitani, C.C.A. Marques, D. Marucci \& M. BRITO, 1992. Microbiotas de Aedes albopictus na região do Vale do Paraíba, Estado de São Paulo, Brasil. Revista Saúde Pública, 26: 108-118.

Gomes, A.C. \& G.R.A.M MARQUES, 1988. Encontro de criadouro natural de Aedes (Stegomyia) albopictus (Skuse), no Estado de São Paulo, Brasil. Revista Saúde Pública, 22: 245.

Gomes, A.C., M.D. Bitencourt, D. Natal, P.L.S Pinto, L.F. Mucci, M.B. Paula, P.R. Urbinatti \& J.M.S. Barata, 1999. Aedes albopictus em área rural do Brasil e implicações na transmissão de febre amarela silvestre. Revista Saúde Pública, 33: 95-97.

Gonçalves, K.S. \& M.C. Messias, 2008. Ocorrência de Aedes (Stegomyia) aegypti (Linnaeus, 1762) (Insecta, Diptera, Culicidae) em bromélias, no município do Rio de Janeiro (Rio de Janeiro, Brasil). Biota Neotropica, 8: 235-237.

Hawley, W.A., 1988. The biology of Ae. albopictus. Journal American Mosquito Control Association, 1: 1-40.

IBGE, 1997. Disponível na internet: <http://www.ibge.gov.br/ home/estatistica/populacao $>$, acesso em 23 de setembro de 2008.

Lopes, J., M.A.N. Silva, A.M. Borsato, V.D.R.B. Oliveira \& F.J.A. Oliveira, 1993. Aedes (Stegomyia) aegypti L. e a culicídeos fauna associada em área urbana da região Sul, Brasil. Revista de Saúde Pública, 27: 326 - 333.

Lourenço-de Oliveira, R., M. G. Castro, M.A.H., Braks \& L. P. Lounibos, 2004. The invasion of urban forest by dengue vectors in Rio de Janeiro. J. Vector Ecology, 29: 94-100.

Miller, B.R. \& M.E. Ballinger, 1998. Aedes albopictus mosquitoes introduced into Brazil: vector competence for yellow fever and dengue viruses. Transactions of the Royal Society of Tropical Medicine and Hygiene, 82: 476-7.

Ministério da Saúde/Fundação Nacional de Saúde/Secretaria Executiva do Plano Diretor de Erradicação do Aedes aegypti do Brasil. Manual de Normas Técnicas. PEAa: Brasília, 1998.

Moore, C.G., C.J. Mitchell, 1997. Aedes albopictus in the United State: ten-year presence and public health implications. Emerging Infec Dis., 3: 1- 8.

\section{Como citar este artigo:}

Barbosa, P.R.M., W.C. Rodrigues \& M.M.O. Cabral, 2010. Incidência das Formas Imaturas de Aedes albopictus (Skuse) e Aedes aegypti (Linnaeus) no Município de Miguel Pereira, RJ, Brasil. EntomoBrasilis, 3(2): 55-58. www.periodico.ebras.bio.br/ojs
O’Meara, G.F., J.R., A.D. Gettman \& J. P. Cuda, 1995. Spread of Aedes albopictus and decline of Ae. aegypti (Diptera: Culicidae) in Florida. Journal Medical Entomology, 32: 55462.

Santos, R. L. C., 2003. Atualização da distribuição de Aedes albopictus no Brasil (1997-2002). Revista Saúde Pública, 37 (5): 671-673.

Serufo, J.C., H.M. Oca, V.A. Tavares, A.M. Souza, R.V. Rosa, M.C. Jamal, J.R. Lemos, M.A. Oliveira, R.M.R Nogueira \& H.G. Schatzmayr, 1993. Isolation of dengue vírus type 1 from larvae of Aedes albopictus in Campos Altos City, State of Minas Gerais, Brazil. Mem. Inst. Oswaldo Cruz, 88 (3): 503 -504 .

Sharma V.P. \& A. Srivastava, 1997. Role of geographic information system in malaria control. Indian Journal of Medical Research, 106:198-204.

Soares, V.A.R.C., W.C. Rodrigues \& M.M.O. Cabral, 2008. Estudo de Áreas e Depósitos Preferenciais de Aedes albopictus (Skuse, 1894) e Aedes aegypti (Linnaeus, 1762) no Município de Paracambi - Rio De Janeiro, Brasil. EntomoBrasilis, 1: 6368.

Suwonkerd, W., Y. Tsuda, M. TAKAGI \& Y. WADA, 1996. Seasonal occurrence of Ae. albopictus in use tires in 19921994, Chiangmai, Thailand. Trop Med., 38:101-5.

Taveira, L.A., L.R. Fontes, D. NATAL, 2001. Manual de diretrizes e procedimentos no controle do Aedes aegypti. Prefeitura Municipal de Ribeirão Preto, 6op.

Urbinatti, P.R., 2004. Observação ecológica do Aedes albopictus em área de proteção ambiental e urbana da periferia na grande São Paulo. Tese de Doutorado. 2004. Faculdade de Saúde Pública da Universidade de São Paulo. Disponível na internet: <http://www.teses.usp.br/teses/disponiveis/6/6132/tde04032005-091946>.

Recebido em: 21/03/2010

Aceito em: 08/07/2010

$* * * * * * * * * * * * *$

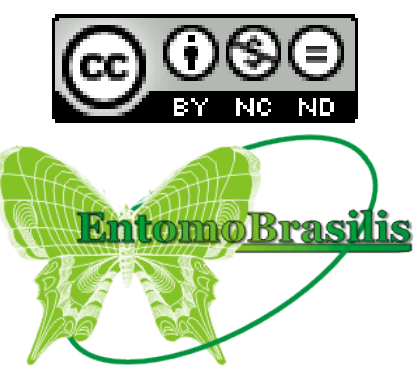

